\title{
Heating without Pollution: The Flameless Burner
}

\section{Fraunhofer Gesellschaft, Munich}

Still an elusive goal for car engines, but now within our grasp for domestic and interior heating: this is combustion without emission of noxious gases. The catalytic gas burner is about to make a breakthrough.

High ozone levels, summer smog in cities, forest depletion, the threat of irreversible changes in the climate: a drastic reduction in atmospheric pollution is the only answer. Critics point their fingers at cars and power stations, overlooking the fact that a third of our energy consumption with the resulting emission may be ascribed to domestic heating. Modern technology offers astonishing potential savings in this field, yet in practice few heating systems are optimized. Furthermore, older systems are often not properly serviced or adjusted.

New laws in Germany will force houseowners to modernise their boilers. The laws on small-scale combustion equipment, which took effect three years ago, were made more stringent from 1 . November 1996. Many older systems will not be able to comply with them; this could apply to some 1.5 million buildings in Germany with heating systems over 15 years old. Since the limiting values will probably be reduced still further in 1999, manufacturers can anticipate a huge market for low-emission burners.

When fossil fuels burn, they produce exhaust gases. Besides the ubiquitous carbon dioxide, which contributes to the greenhouse effect, there are smaller amounts of poisonous carbon monoxide ( $\mathrm{CO}$ ), various nitrogen oxides (NOx), and other airborne pollutants. Rather than poison the inhabitants of the house, the gases are led out through a chimney to pollute the atmosphere. For example, nitrogen oxides play a decisive role in the formation of ozone at ground level.

Modern technology not only aims to increase the efficiency of heating plants but also to drastically reduce the emission of harmful gases. Of course it would be best to prevent the production of toxic gases in the first place: by choosing a completely

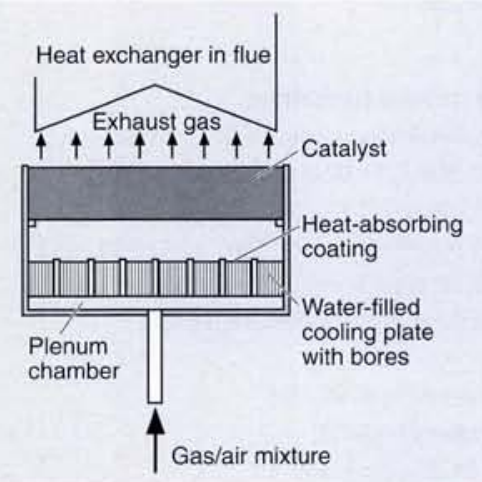

Fig. 1. Schematic representation of the catalytic burner different combustion method - flameless catalytic combustion. The non plus ultra of modern heating technology is an innovative burner in which a mixture of air and natural gas is not burnt with a flame, but oxidised using a catalyst. The Fraunhofer Institute for Solar Energy Systems (ISE) in Freiburg has developed a flameless burner now undergoing practical trials in a pilot scheme.

Conventional burners suffer from the disadvantage that temperatures above $1200^{\circ} \mathrm{C}$ and reactions in the flame front generate nitrogen oxide and other harmful substances. Catalytic combustion tackles the problem at its origin: there is no open flame and the temperature is kept below $1000^{\circ} \mathrm{C}$.

The principle goes back to J. W. Döbereiner, chemistry professor in Jena, who in 1823 recognised the catalytic effect of platinum metals and employed it to develop a cigar lighter. Platinum accelerates the combustion process without itself being consumed in the process. It lowers the combustion temperature: hydrogen ignites catalytically at room temperature. For methane, natural gas' main constituent, rather higher temperatures are necessary for combustion (from $250-400^{\circ} \mathrm{C}$ ), so preheating is required. The catalytic converter in a car cannot start cleaning the exhaust until it reaches its operating temperature.

Much thought has been given to the catalytic treatment of exhaust gases from cars and power stations, but little has been done about domestic systems. Catalytic converters are designed for complete conversion of relatively low concentrations of hydrocarbons in exhaust or flue gases. High power densities, as demanded of a heating plant, require a correspondingly high concentration of fuel - the high thermal load would destroy the catalyst. This is the essential problem facing the scientists. But at ISE they have succeeded in combining a normal commercial honey-comb structure with a cooling device such that the catalytic burner produces high powers yet can still function reliably.

Once the catalyst has been preheated, stable and almost $100 \%$ complete combustion begins in the fine honeycomb structure. The resultant heat is transferred to the flue gas and by radiation to the cooling plate below. The water circulating in this plate is heated; at the same time the design ensures that more heat is extracted from the catalyst as the rate of combustion increases. This self-regulation process prevents the material overheating (Fig. 1).

The ISE fitted a prototype catalytic burner with $10 \mathrm{~kW}$ output to a normal commercial boiler. The result of several hundered hours of operation were

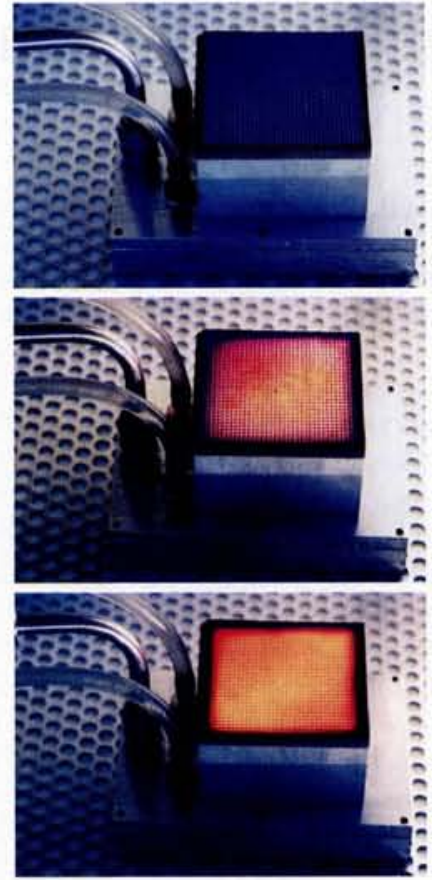

$1-k W$ burner module preheat phase with low-NOx flame burner

$1-\mathrm{kW}$ burner module transition from flame to catalytic combustion

$1-\mathrm{kW}$ burner module operating in catalytic mode most encouraging: the $\mathrm{NOx}$ values in the flue gas were only about $0.5 \mathrm{mg} \mathrm{kWh}-1$ (conventional flame burners produce $100-200$ times as much, depending on design). The $\mathrm{CO}$ and unburnt methane emissions were both under $5 \mathrm{mg} \mathrm{kWh}-1$, close to the detection limit.

The low-emission boiler was recently installed in a technology centre in east Germany. Not only is this pilot plant a good advertisement for an environmentfriendly heating system, but it is providing researchers with valuable experience of the new technology under running conditions. Further investigation by the ISE has shown that the catalytic burner principle can be varied for use in other applications, such as heating tents, caravans, or portable cabins on building sites. Or an air-conditioning plant may even be powered. Another application might be catalytically accelerated chemical reactions, e.g. in reforming natural gas.

The flameless burner will set new standards in heating engineering and pave the way to zeroemission energy of the future, not just in a clean but also fast and easy implementation. It could make a valuable contribution to the reduction of atmospheric pollution in cities to tolerable levels.

Fraunhofer Gesellschaft, Presse- und Öffentlichkeitsarbeit

\section{For further information, please contact:}

Dr. Alexander Schuler

Fraunhofer-Institut für Solare Energiesysteme ISE Oltmansstrasse 5, D-79100 Freiburg

Tel: +49-761-4588-213;

Fax: $+49-761-4588-320$ 


\section{Coating Increases the Life of Surgical Implants}

\section{Reports from the Netherlands}

Implanting ear drainage tubes, pacemakers or artificial joints is something which surgeons nowadays do on an everyday basis. However, almost $5-40 \%$ of such operations are a failure, partly because the body's tissue does not adhere properly to the implant. A Dutch team supervised by Prof. J. Jansen of the University of Nijmegen has developed a simple method of providing metal implants with a structured ceramic coating. This appears to have a positive influence on the way the bone heals. In addition, the implants are tolerated better by the body and their clinical life is longer. The project is being carried out with support from the Technology Foundation of the Netherlands Organization for Scientific Research (NWO).

When replacing bone or teeth, surgeons prefer to use prostheses made of calcium phosphate ceramic material. The surface of this substance makes an active bond with the surrounding bone, but the material is rather brittle. In the case of artificial hips or tooth implants, which are subject to major stresses, the preferred material is metal with a thin layer of ceramic calcium phosphate. But manufacturing this is not a simple matter.

Prof. Jansen's team has spent the past 5 years developing a technique to apply the thin ceramic layers required by "bombarding" the so-called ceramic target with ions of the inert gas argon in a vacuum. The ions "knock off" particles of the ceramic material which then adhere to the metal of the prosthesis. The result is a coating which is extremely thin, namely between 1 and 10 microns. The structure of the coating can also be adapted to the particular purpose for which the prosthesis is required.

Like its chemical composition, the structure of the implant's surface influences the reaction of the surrounding tissue. The Nijmegen study has also shown that the reaction of the living cells around an artificial implant can be most effectively influenced if there are 1-5 micron wide grooves in the surface of the implant, This causes the cells of the tissue which subsequently attaches itself to grow in a preferred, single direction. If the grooves are made wider, the cells grow in all directions, reducing both the level of adhesion and the clinical life of the implant. The Dutch researchers hope that by applying the coating as surface micro-texturing, they may be able to increase the life of an implant by 5 to 10 years. A Swedish company has already acquired the manufacturing rights to the new "Nijmegen coating".

\section{Further information:}

Prof. J.A. Jansen, University of Nijmegen

Tel: +3124361 4921; Fax: +31243541971

Email:j.jansen@dent.kun.nl

\section{Spectra-Physics Lasers offers Pro Series Nd: YAG Laser}

\section{Product News from Spectra-Physics}

Spectra-Physics Lasers (SPL) introduces the Quanta-Ray Pro Nd:YAG laser, offering repetition rates from $10-50 \mathrm{~Hz}$ and pulse energies in excess of $2500 \mathrm{~mJ}$.

The Pro series has been designed for a variety of applications, including pumping of OPOs, multipass and regenerative amplifiers, together with other demanding roles requiring stable output performance at $1064 \mathrm{~nm}, 532 \mathrm{~nm}$ or in the UV.

This new laser provides near and far-field spatial beam profiles which are tailored to different applications, while maintaining high energy extraction, alignment stability and excellent passive beam pointing and divergence characteristics.

\section{- and Offers Accessory to Improve Nd:YAG Laser Performance}

Spectra-Physics Lasers introduces BeamLok, an accessory designed for its new Quanta-Ray Pro Series $\mathrm{Nd}$ : YAG lasers. This option enhances laser system performance by offering advanced beam pointing with active control and divergence reduction, countering the effects of lamp aging.

BeamLok incorporates three active detection and correction systems: a far-field detector, corrective mirror and D-Lok sensor. All three are integrated by a dedicated microprocessor-based controller to provide complete feedback control.

The resulting system has faster warm-up times, typically under $15 \mathrm{~min}$., longer flashlamp lifetimes with more productive output over time, and exceptional beam pointing stability resulting in fewer downstream alignments. The monitoring and control system is self-contained within the Nd: YAG laser. The stand-alone controller has a complete graphical interface to allow easy access to all system parameters. RS-232/ IEEE computability enables BeamLok to be incorporated into existing automated systems.

The BeamLok system has been developed for today's demanding applications including pumping $\mathrm{OPOS}$, regenerative and multipass amplifiers, and applications which require stable beam properties in the fundamental harmonics. The passive stability of Quanta-Ray Nd:YAG lasers is well known and the BeamLok active control system further enhances performance.
All Quanta-Ray Nd:YAG lasers feature: gold elliptical reflectors for optimum mode properties, sealed beam paths for system integrity, thermal depolarization compensation for improved beam quality and high-damage-threshold optics for added reliability.

To further enhance the performance of the standard Pro Series laser, SPL offers its new BeamLok option (see below), an accessory that provides even greater beam pointing capabilities and maintains lower beam divergence over the life of the lamp. Moreover, BeamLok enables faster warm-up times, promotes more stable system performance and prolongs performance over the flashlamp lifetime.

Founded in 1961, Spectra-Physics Lasers is a leading designer and supplier of lasers to the scientific and industrial communities. The company offers a diverse range of products backed by the largest worldwide sales and service support organization in the laser industry.

For more information:

Adrian Rowland, Spectra-Physics Lasers Inc., P.0. Box 7013, Mountain View,

CA 94039-7013.

Tel: +1-415-966-5500; Fax: +1-415-969-5921 email: arowland@splasers.com website: http://www.splasers.com. or

Spectra-Physics GmbH

Siemensstrasse 20

D-64289 Darmstadt

Tel: +49-6151-708-224; Fax: +49-6151-79102 